\title{
The Research on Ecological Chain Structure of High Intelligence Work in the Big Data Era
}

\author{
Yin Fengchun ${ }^{1,2, *} \&$ Yuan Wenli ${ }^{1}$ \\ ${ }^{1}$ Yancheng Teachers University, Yancheng, Jiangsu 224051, China \\ ${ }^{2}$ School of Business, Hohai University, Nanjing, Jiangsu 210098, China \\ *Correspondence: Yancheng Teachers University, Yancheng, China. E-mail: hobbyc@163.com
}

Received: October 9, 2017 Accepted: October 20, 2017 Online Published: October 28, 2017

doi:10.5430/mos.v4n4p29 URL: https://doi.org/10.5430/mos.v4n4p29

\begin{abstract}
In the big data era, highly intelligent job embeddedness promotes organizational ecology, chain value, and development potential. This paper analyzes the nature and representation of intellectual work embedded, and explores the operation mode of big data era intellectual work embedded in the ecological chain, based on cooperative competition ecological chain, value chain, proliferation of ecological symbiotic ecosystem, ecological equilibrium chain, information aggregation structure driven ecological chain, optimizing the environment of the ecological chain.
\end{abstract}

Keywords: big data; high-end talents; job embeddedness; ecological chain; structure

\section{Introduction}

In the era of big data, the degree and level of high intelligence work embeddedness are positively related to the operation level of organizational ecology chain. The higher the degree of embeddedness, the greater the potential motivation, and the higher the value level of the ecological chain embedded in the higher intellectual work. Big data is a collection of data that contains a lot of information, high-speed output, diversified forms, low value density, and real performance. High intelligence refers to those who master systematic theory knowledge or have higher technology and can make important contributions to material civilization, spiritual civilization, political civilization and ecological civilization. Job embeddedness is the analysis of the organizational viscosity of talents from the three dimensions of connection, matching and sacrifice. Work with the ecological chain operation embedding problem to the point of view of high intelligence, in order to show the ecological chain operation form, from the analysis of multiple factors and characteristics, on how to improve the intellectual organization in the ecological chain of job embeddedness, has important significance and practical needs.

\section{The Connotation, Characteristics and Influencing Factors of Embedded Eco Chain in the Era Of Big Data}

\subsection{The Connotation of High Intelligence Work Embedded In Ecological Chain in the Era of Big Data}

In the era of big data, high intelligence work is embedded in the ecological chain. In the era of big data, the process and mode of embedding ecological chain of highly intelligent individuals or groups of work are analyzed from the following three points:

Quantity and quality of eco chain embedded in high intellectual work. The quantity and quality of intellectual work embedded in ecological chain refers to the quantity and quality of high intelligence tissues in the ecological chain. Understand quantity and quality from the angle of human resource. "Quantity" refers to the number of talents who constitute part of the labor force population, and the distribution of "quantity" has a positive correlation with the level of regional economic development; "Quality" is from knowledge, work skills, creative ability, the ability to adapt to the job, organizational management level to examine the input-output ratio of human resources. The higher the ratio, the higher the quality, vice versa. High intelligence belongs to the scarce human resources, and every high intelligence in the organizational ecological chain is of great value. High intelligence quantity and quality is lower than organization demand, the development speed of organization is slow, and finally gets into trouble. The increase 
in the amount of ecological chain can be considered from these aspects: First, the introduction of high intelligence to join the organization, and reasonable matching: to provide high intelligence, attractive jobs, direct recruitment; Create an open and free organizational culture and attract high intelligence. Second, keep the original high aspiration of the organization intact and increase the sacrifice: To give high intelligence, broad career development space, to make full use of intellectual values, can be promoted in the position; The establishment of a smooth, high intelligence assistance mechanism, in a timely manner to find high intellectual difficulties encountered in work life, and help them solve the problem. Third, develop and train excellent members of the organization to become highly intelligent and establish connections: Identify members of the organization and select potential talents for training; The organization regularly provides opportunities for learning and communication within and outside the organization, so that talented people will have the opportunity to enhance their ability to become highly intelligent. Fourth, improve the quality of the ecological chain: Study of professional knowledge, encourage members of on-the-job learning, provide places; work skill, provide independent jobs, so that members can complete a work alone; the cultivation of creative ability, to create an open organization culture, the members of the organization to support multi path to solve the problem; post fit improved distribution post and post selection and combination of independent, so that members have the right to choose jobs; training, organization and management ability of the moderate decentralization, let the organization to participate in the management of high intelligence.

The attributes and behaviors of high intelligence work embedded in ecological chain. Attributes affect behavior, behavior, expression, attributes. An attribute is an abstract concept within. Physically strong, intelligent super, strong psychological quality, wide knowledge, innovative learning ability, usually high intellectual attributes. Behavior refers to the external manifestation of high intellectual attributes. The embedded nature of high intelligence work refers to the integration of highly intelligent individuals into the organizational ecology chain, becoming part of the ecological chain, and mutualism with other parts of the ecological chain. The behavior for the high intelligence into the work of the organization, according to the similarity, intellectual and organizational environment; high intelligence in the working process and organization of large and small, the number of uncertain connection; High intelligence once left the organization will face the sacrifice, the sacrifice may be economic benefits, may also be psychological aspects.

The attributes and behaviors of high intelligence work embedded in ecological chain. Attributes affect behavior, behavior, expression, attributes. An attribute is an abstract concept within. Physically strong, intelligent super, strong psychological quality, wide knowledge, innovative learning ability, usually high intellectual attributes. Behavior refers to the external manifestation of high intellectual attributes. The embedded nature of high intelligence work refers to the integration of highly intelligent individuals into the organizational ecology chain, becoming part of the ecological chain, and mutualism with other parts of the ecological chain. The behavior for the high intelligence into the work of the organization, according to the similarity, intellectual and organizational environment; high intelligence in the working process and organization of large and small, the number of uncertain connection; High intelligence once left the organization will face the sacrifice, the sacrifice may be economic benefits, may also be psychological aspects.

\subsection{Analysis of Embedded Eco Chain in High Intelligence Work in Big Data Era}

In the era of big data, the characteristics of eco - chain construction embedded in high intelligence work are mainly:

The cyclist of high intelligence work embeddedness. The oceans, the land and the space layer each have a circulation system and form a large circulatory system. Example: water evaporates from the ocean into the space layer. Clouds accumulate in the space layer. The rain falls into the land. The rain collects the rivers and flows into the ocean. The process goes round and round. The cycle of high intelligence job embeddedness means that the process of embedding high intelligence work is cyclical and endless. A cycle of experience, trauma, self-repair, growth, and other processes that are embedded in the ecological chain. Establish connection in the early stage of high intelligence, high intelligence into the organization are not very understanding of all aspects of the organization system, culture, personnel, to adapt to various trauma; mid high intellectual adjustment ability through their strong self-repair quickly into the organization; later, high intelligence is fully integrated into the ecological chain, the rapid growth of the ecological chain in the organizational culture and job skills to realize their own ability. In the process of setting up a link, the growth process of high intelligence cycle, makes the degree of job embeddedness more deeply. Organizations are built by people, each with a systematic cycle and a large circulatory system, that is, the ecological chain of organization.

Clustering of highly intelligent job embeddedness. The process of separating sets of different objects into multiple classes consisting of objects of similarity is called clustering. Clustering of highly intelligent job embeddedness 
means that high intelligence also follows classification characteristics when clustering. Like attracts like. Birds of a feather flock together. High intelligence covers a wide range of professional skills, such as: enterprise management personnel, high-tech research and development personnel, professional skills personnel. In the process of job embeddedness, high intelligence is divided into different types of high intelligence because of their respective advantages and skills, and similar types of high intelligence are naturally collected in the ecological chain. In enterprise management, the same type of high intelligence usually comes together in the same department, which is the most common clustering phenomenon. When gathering high intelligence of the same nature, we can complement each other by adding blind areas of cognition, expanding knowledge, colliding ideas, generating innovative thinking and forming a healthy competition. The value of clustering intelligence is much higher than that of a single high intelligence, and in this process, high intelligence itself has realized value added.

Synergy of high intelligence work embeddedness. The synergy of high intelligence work embeddedness refers to the characteristics of coordination and coordination among organizations in order to achieve common goals. In the process of job embeddedness, intellectual and organizational components to establish connections to form a network like structure through a connection with other intellectual part of the flow of information sharing, the internal ecological chain collaboration become possible. A high intellectual synergy state in the work embedded, can give full play to their talents and resources with the help of connectionist networks, by connecting with other network dispatching organization manpower material and financial resources to coordinate, eventually greatly improve the ability to organize the ecological chain complete organizational goals.

Robustness of high intelligence job embeddedness. Robustness is the transliteration of Robust, that is, "robust" and "strong". It is the key to a system that does not crash in cases of unusual and dangerous conditions. The robustness of embedded work with high intelligence refers to the ability of high intelligence to remain stable in the process of job embeddedness without adverse external influences. The improvement of robustness depends on the comprehensive ability improvement of high intelligence itself and the optimization of ecological chain operation mode. High intelligence has a strong sense of competition, in the work, through constant changes, interpersonal relations and environmental adjustment, repeatedly hone professional skills, update knowledge storage, comprehensive promotion of comprehensive ability. The ecological chain in the working path of high MI, select the optimal path between high intelligence and high effective intellectual connection, optimal path through intellectual repeated establishment, operation optimization and overall ecological chain. Enhance robustness in the growth of high intelligence and organizations.

\subsection{Analysis of Influencing Factors of Eco Chain Embedded in High Intelligence Work in the Big Data Age}

The main factors influencing the ecological embeddedness of high intelligence work are:

High intelligence embeddedness, role dynamics, and multiple niche effects. The niche refers to the natural environment, each specific location has different species, special structure, it depends on the relationship between the physiological and behavioral activities and other creatures, so it has its unique niche. High intelligence refers to the dynamic role of the high intelligence in the process of job embeddedness, because of the face of people, things and things continue to change, the high intelligence ideas and working methods are also mature. The reasons for this phenomenon are that the job embeddedness process has multiple niches: First, the ability of high intelligence itself is a complex highly, can handle different types of work; second, high intelligence in the process of job embeddedness, contact and fusion of diverse skills; third, high intelligence and multi role dynamic niche, established the high intelligence and ecological chain and multi direction connection[15]. Promote intellectual job embeddedness. In the process, High intelligence realized the rapid growth of his comprehensive ability, and made the ecological chain run more efficiently and flexibly.

The influence of high intelligence on the changeable information and the ecological field of activity. Information is changeable, which means that information changes at different times and under different circumstances. The field refers to the space, and the activity ecological field refers to the flexible ecological space possessed by high intelligence in the ecological chain of the organization. Information is changing because of changes in environment, time, and flow. The dimension of the information scope increases with the change of interest, and the corresponding processing dimension show a positive proportion. High intelligence in the ecological chain occupies multiple niches, can make high intelligence flexible processing of changeable information, in the dynamic transfer process, formed a flexible ecological field. The establishment of ecological field need to have the following conditions: first, the establishment of an open and inclusive organizational culture, is to determine the key problems existing in the ecological field activities; second, made in support of the ecological niche ecological chain, intellectual ecological field of the activity will be exposed to more nodes in the ecological chain. 
High intelligence embedded in information nodes and cluster ecology can influence. The information node in the ecological chain is the hub of organizing information channels, which has great influence. High intelligence embedded in information nodes has the following advantages: First, high intelligence can enlarge the value, improve ecological efficiency ecological chain; second, High intelligence has deep expertise and strong execution, work to be able to arrange the ecological chain of other individuals, and proposes the reasonable problem case; Third, the professional accomplishment of high intelligence and dedication has a positive impact on other individuals in the ecological chain, and improves the ecological energy of each individual in a number of ways..

\section{Construction of Ecological Chain Embedded in High Intelligence Work in the Big Data Era}

By analyzing the evolution process of the eco chain embedded in the high intelligence work and the structure of the eco chain of high intelligence, the evolution and structure of the embedded eco chain of the high intelligence work in the big data era are studied:

\subsection{Evolution of Embedded Eco Chain in High Intelligence Work in the Big Data Era}

The development of things to follow certain rules, the process of intellectual work embedded has experienced an evolutionary process, which are germination stage(GS), growth period(GP), stable stage(SS), stagflation period(SP), restructuring period $(\mathrm{RP})$ :

Evolution of ecological chain

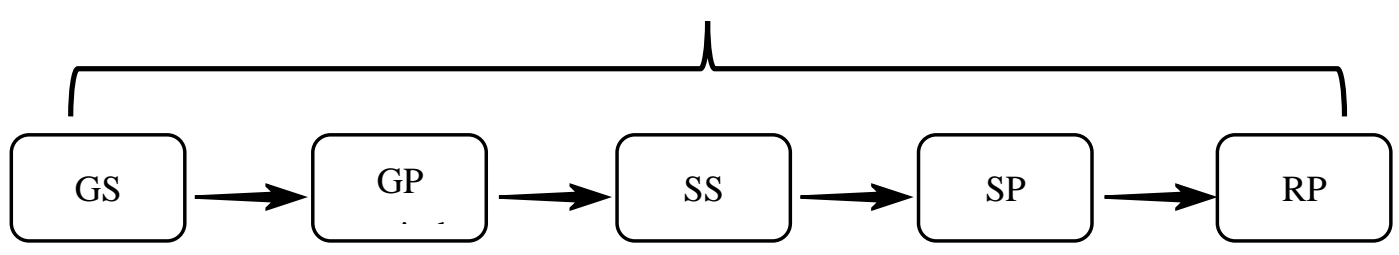

Figure 1. Ecological Chain Evolution

High intellectual work is embedded in the embryonic period of ecology. The embryonic stage of high intelligence work embeddedness is that high intelligence enters the organizational ecological chain at the initial stage. High intelligence must be fully aware of their strengths and weaknesses, and judge and fit the position of the formation of matching. This is a process in which High intelligence understands the integration of the ecological chain. High intelligence should set up the correct communication method for the superiors, peers and subordinates in the ecological chain of the organization; Have a clear understanding of the ecological chain in the organization of rules and regulations or internal to the organization, in common; ecological chain problems, development trend and preliminary planning of their organization in the ecological chain development orientation. The embryonic stage of the eco organization embedded in the highly intelligent work is a very delicate period. It is the process of the formal contact with the ecological chain of the high intelligence organizations, and also the process of organizing deep understanding of the high intellectual. During the germination period, organizations need to provide more help and tolerance for higher intellectual ability, which is conducive to high intellectual work Embeddedness and is rooted in the organizational ecology chain.

High intellectual work is embedded in the ecological growth phase. The growth period of high intellectual work embeddedness is the period of rapid development of intellectual organizations. In adapting to the organizational environment, High intelligence absorbs the essence of culture in the organization and develops rapidly, and feeds the result of growth to the ecological chain. The growth of high intelligence can also promote the growth of other members of the ecological chain of the organization, and promote the formation of a healthy competition atmosphere in the organizational ecological chain. At the same time, High intelligence has accumulated rich experience in the growth period. According to the specific circumstances of the ecological chain of the organization, he uses his own innovative thinking and specialized knowledge to solve the problems existing in the optimization of the ecological chain of the organization. During the period of growth, the ecological field of high intelligence activities provides enough space to display their talents and promote the healthy development of the ecological chain of the organization. 
High intelligence work is embedded in the ecological stable period. High intelligence work embedded in the ecological stable period means that high intelligence has been fully adapted to integrate into the ecological chain. In the period of stability, high intelligence has been able to play a role in the ecological chain, culture, identity and participate in the organization of the ecological chain and the ecological chain into fully familiar with the operation mode, and other intellectual ecological chain to establish a stable social relationship. During this period, high intelligence's own development in the organization and the promotion speed of the development of the ecological chain of the organization, compared to the growth period down, and entered a stable stage of development.

High intellectual work is embedded in the ecological stagflation period. High-end job embeddedness refers to ecological stagnation, in the development of long time, high intelligence in organization on organizational growth and ecological chain to promote the stagnant period. This may include: first, high intelligence in the early to greatly promote the progress of the ecological chain, ecological chain into the higher stage of development, the current high wisdom cannot play the role of the second pilot drive; high, chi in the ecological chain in the long-term development and ecological chain completely assimilated and lost the culture of thinking brought by the collision sparks, competition in the stable development in the fuzzy sense.

High intellectual work is embedded in the ecological recombination phase. The ecological reorganization stage of "High intelligence" work refers to the long-term stagnation development of high intelligence and organizational ecology chain, which will lead to the decline of high intellectual value and the collapse of organizations, and need to be re matched and restructured. In the process of restructuring, high intelligence will face the sacrifice of leaving the organization. It includes material sacrifice and emotional sacrifice. Leaving the original chain of high Chi will re-enter the matching process, looking for their own organizational ecology chain. The original eco chain is also looking for high intelligence to match the needs of organizational development. Reorganization is a way to let the high wisdom and revitalized the ecological chain organization.

\subsection{The Structure of Eco Chain}

High intelligence, job embeddedness, collaborative competition, ecological chain. The ecological chain of cooperative competition refers to the opposite process of unity and interdependence, mutual dependence, mutual guidance and mutual transformation between the two sides of the ecological chain. Competition leads to coordination and coordination leads to competition. The individual in the ecological chain due to the development trend cannot see the global ecological chain, for selfishness thinking, will find a development direction for spontaneous ontology, other individual cooperation and competition continued in the state and the ecological chain. In the process of embedding eco competition into the eco competitive chain, it is necessary to quickly adapt to the eco chain operation mode of cooperation and competition. Must play high intelligence, have strong competition ability, can occupy a place in zoology chain. High intelligence, by analyzing the situation in which he is, calculates his own strengths and weaknesses, and needs to find the right individuals in the ecological chain. Highly intelligent job embeddedness will increase the intensity of competition in the ecological chain and bring impact and vitality to the ecological chain. Let other members of the ecological chain, in this process, maximize their potential, enhance their ability, thereby bringing the entire ecological chain optimization. Cooperation and competition coexist in a collaborative ecological chain, and high intelligence job embeddedness promotes synergy and promotes competition.

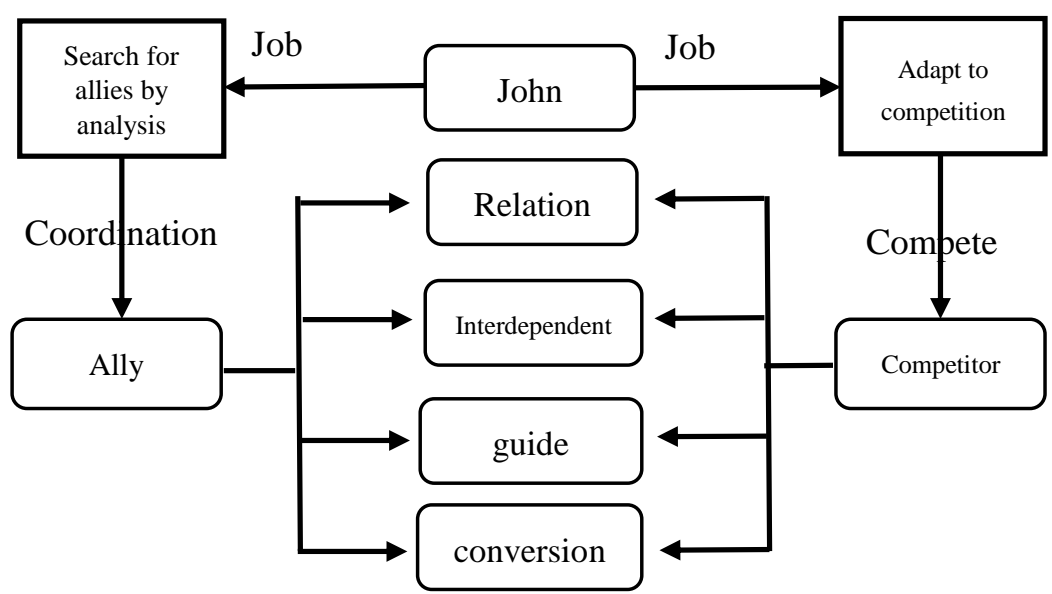

Figure 2. Job Embeddedness Collaborative Competition Eco Chain 
High intelligence work, embedded value, proliferation, ecological chain. Value added ecological chain refers to the multiplication of assets through management or operation activities. In this process, although the cost of high intelligence itself is greater than the cost of ordinary human resources, but the value of proliferation in quantity and quality are far more than ordinary human resources. Because has the ability of high intelligence is compound: first, the high complex capability allows high intelligence to play a greater value in the process of embedding, improve work efficiency and achieve the purpose of improving organizational performance; Second, bring this high value proliferation ability of composite will be more comprehensive, not only is the proliferation of organizational ecology chain assets visible, as well as the organization of the ecological chain of intangible assets such as proliferation, proliferation and the ability of the team technical research and development capabilities of value-added and social benefits of the proliferation etc..

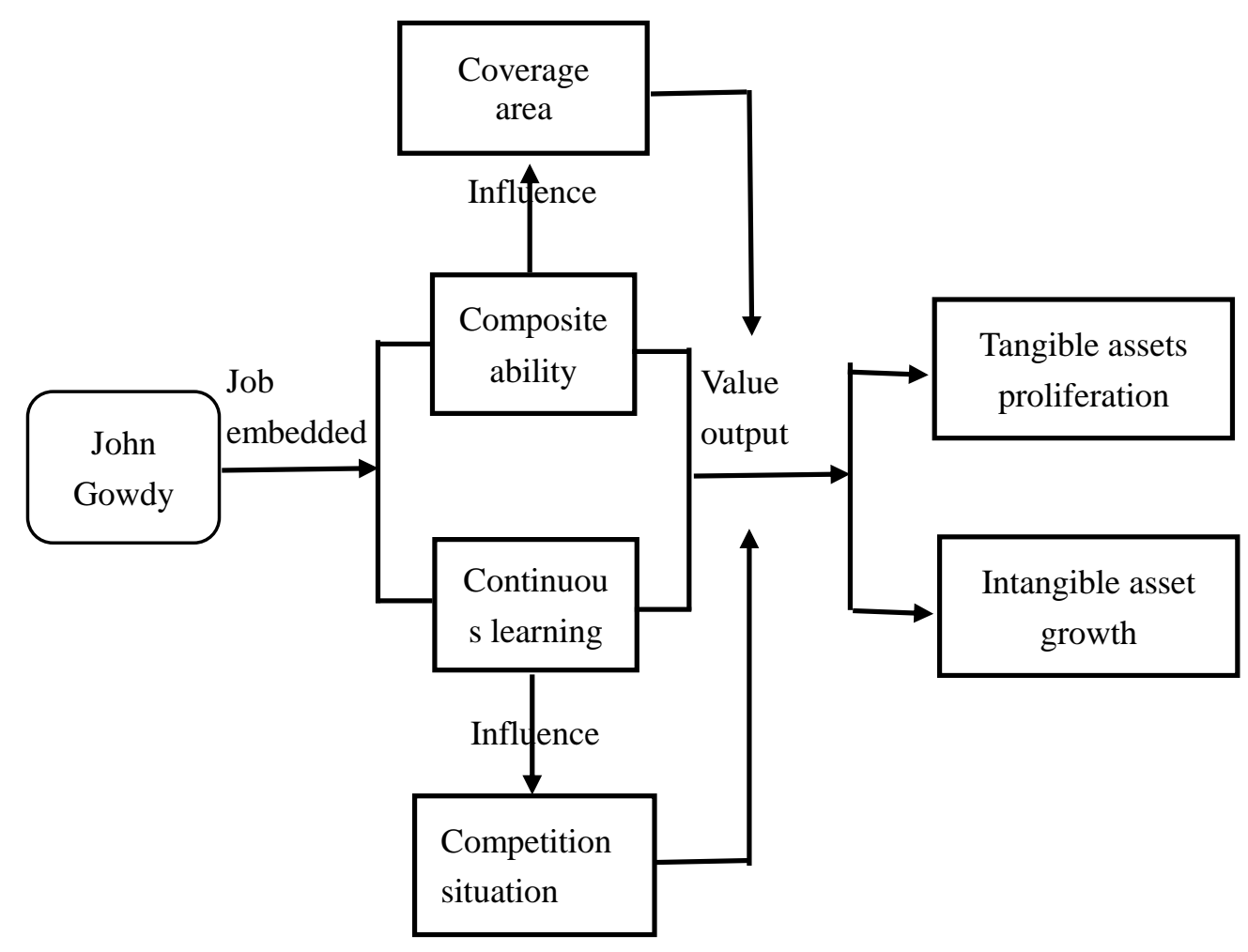

Figure 3. Job Embeddedness, Value Increase, Ecological Chain

High intelligence work, embedded mutualism, ecological chain. Mutualism ecological chain refers to the ecological chain of the various components of cooperation with each other, and this cooperation is beneficial to each other. High intellectual work is embedded in mutually beneficial symbiosis, and ecological chains inevitably coexist with other components of the ecological chain. Under the premise of fierce competition and rapid change of the external environment, each member of the ecological chain has to cooperate with each other to make the ecological chain develop to the maximum extent. High intelligence takes full advantage of its ability and insights into the ability of other members of the ecological chain to identify patterns that maximize symbiosis through identification and analysis. High intelligence job embeddedness will put this symbiotic ecological chain advantage further show the powerful advantage of high intelligence itself will bring more value to other members of the ecological chain symbiosis such as ideas, technology research and development of flexible and efficient operation mode and principle innovation responsible work attitude and so on. 


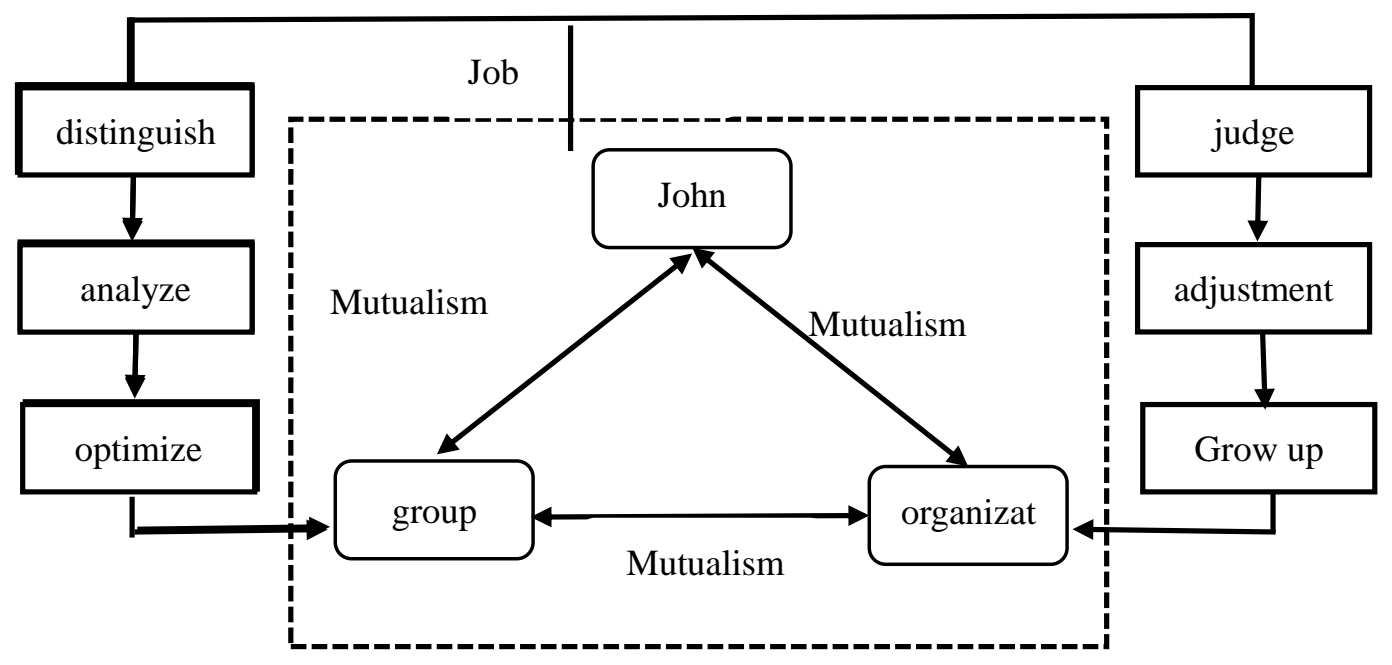

Figure 4. Job Embeddedness Mutualism Ecological Chain

High intelligence, job embeddedness game, equilibrium ecological chain. The game equilibrium ecological chain refers to the existence of the game relationship in each component of the ecological chain. Intellectual work embedded game equilibrium ecological chain will break the initial equilibrium, resulting in a new shock to the entire ecological chain, because in the early work of embedded, high intelligence in the game no matter which party is as strong as foreign aid, to break the situation of well-matched in strength. But in the game equilibrium of the ecological chain, game competition consciousness is very strong, the other side of the game through a variety of ways to enhance one's own competitive ability, the fierce degree of organization in the ecological chain game will be more intense than before, the ecological chain organization will re reach equilibrium situation until high embedded intelligence work after a period of time

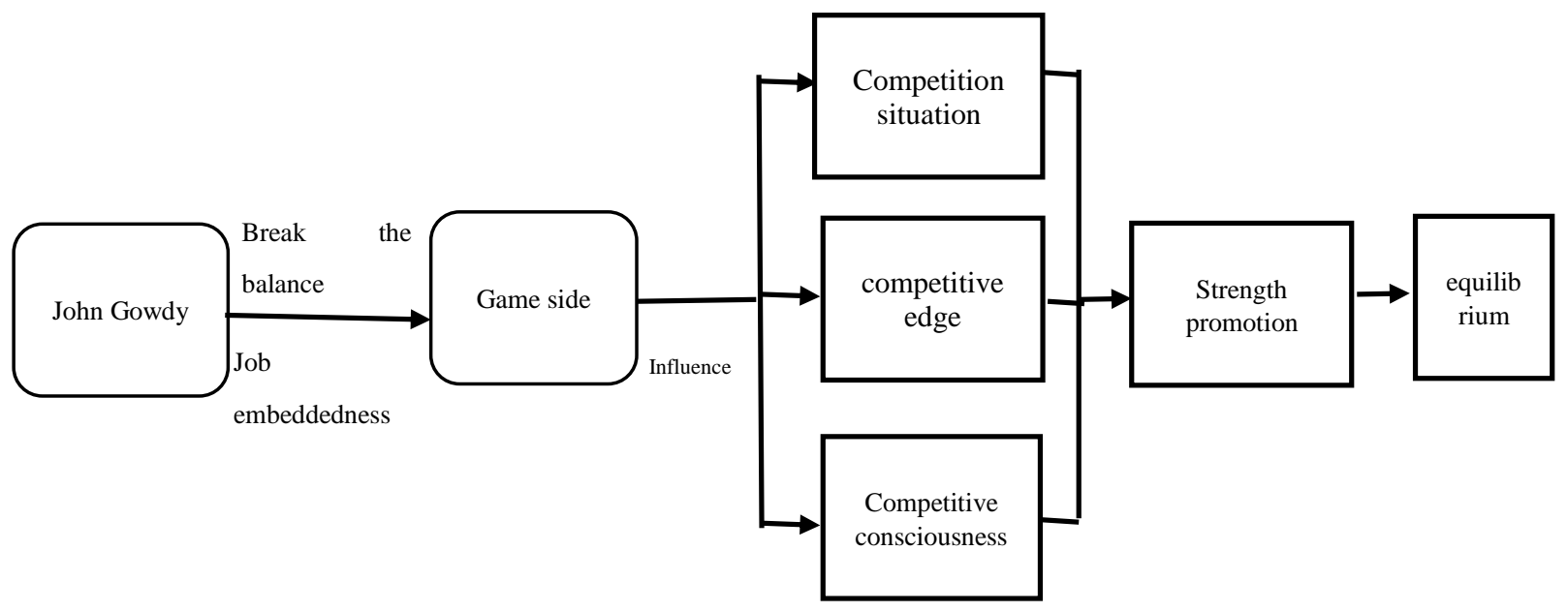

Figure 5. Job Embeddedness, Game Equilibrium, Ecological Chain

High intelligence work, embedded information, cohesion drive ecology. Information aggregation driven ecological chain refers to all kinds of information organization in the ecological chain together, unified cleaning, screening, and summary, and condensed into information ecological chain nodes, the node information as to promote ecological chain operation development impetus. High intelligence is precise in the judgment of information meaning and flexible in dealing with information. High intelligence job embeddedness can improve the driving force from the following aspects: first, the high intelligence itself can directly deal with some emergency information, improve the response speed of ecological chain system; second, system flow high intelligence information 
processing optimization of the original ecosystem, to achieve precise docking data and individual ecological chain; third, high through the establishment of intellectual management system to enhance ecological chain of each individual information and the response speed of execution.

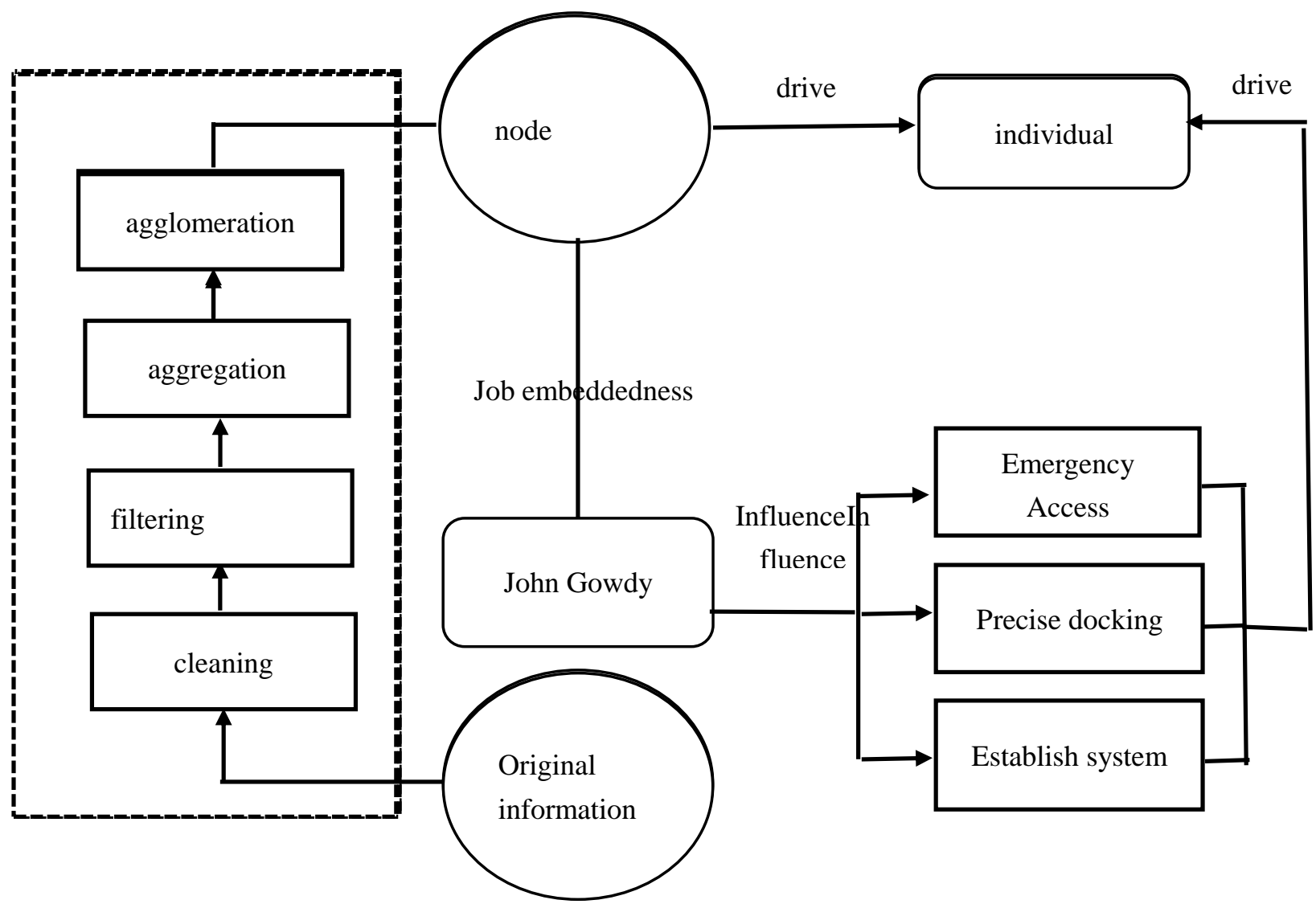

Figure 6. Work Embedding Information to Drive the Eco Chain

High intelligence work, embedded environment, optimization, ecological chain. Environmental optimization ecological chain refers to the ecological chain between high intelligence and ecological chain environment. High intelligence works to embed the environment, optimize the ecological chain, establish links with other individuals in the ecological chain, and form a feedback mechanism with the ecological chain environment. High intelligence directly analyzes the ecological chain environment and optimizes the ecological environment. High intelligence indirectly affects other individuals through their positive energy, which enables other individuals to optimize the ecological chain environment. After the ecological chain environment is optimized, the ecological chain individuals are fed back, and the environment and the individual form a virtuous circle. 


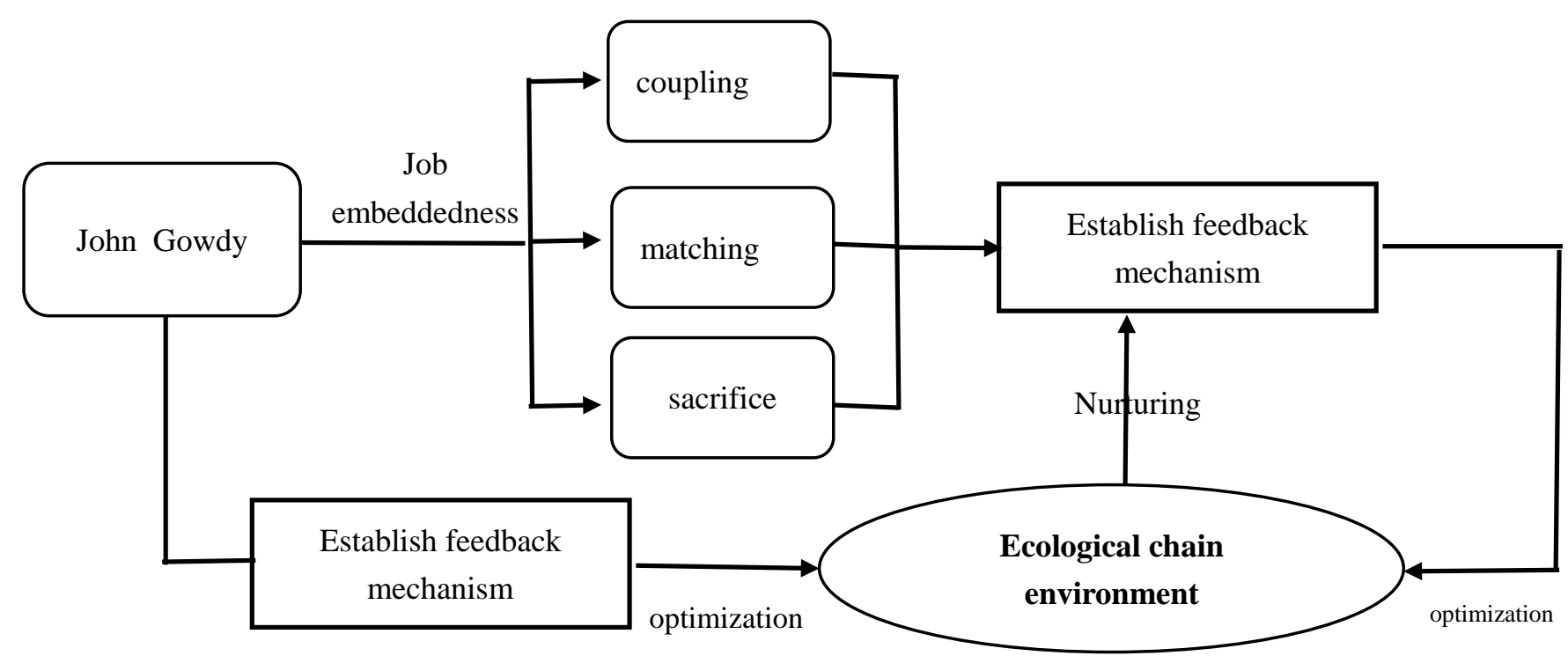

Figure 7. Work Embeddedness, Environment Optimization, Ecological Chain

\section{High Intelligence Work Embedded in the Operation of the Ecological Chain In Big Data Era}

Big data is the main driving force of modern enterprise innovation can create new products or services, improve existing products and services technology level. The use of big data and tools based on big data can greatly promote intelligent work, embedded in the ecological chain of intelligent operation.

\subsection{Simulation Data Guide the Refinement of High Intelligence Work Embedded Collaborative Competition}

The simulated data is static model is knowledge, process knowledge, rules control knowledge, operation logic simulation data is the rule control model of operation model, data processing, data support and drive simulation. Simulation data simulation is used to simulate the possible occurrence of high intelligence in the eco competitive chain of job embededness, and predict the results of collaborative competition in advance, and select the favorable path carefully.

To find out the high degree of organization, the highest wisdom ecological chain collaboration, through the simulation data reflect the result of cooperative competition in the process of guiding direction adjustment, to achieve high intelligence at work embedded in the whole process of fine control, realize the ecological chain to introduce high intellectual value maximization.

\subsection{Cloud Computing to Promote High Intelligence Work, Embedded Value, Proliferation, and Node}

Cloud computing, according to data computing requirements, can be used to idle computer resources on the Internet, similar to networks, servers, storage space, applications, etc., can be attributed to computer resources. Cloud computing is the product of the era of big data burst data, big data era of massive increase the difficulty of the data processing, the development of cloud computing technology to reduce the processing cost of the data by finding the value proliferation organization in the ecological chain of massive data processing, achieve rapid docking process and high value proliferation embedded intelligence work the key of strengthening by intellectual strong management ability, the ecological chain cost every investment can achieve the greatest degree of increase in value through the proliferation point.

\subsection{Intelligent Feedback, Improve High Intelligence Work, Embedded Symbiosis, Networking}

In biology, the more complex the ecological chain, the more species, it shows that the higher the ecological chain, the stronger the robustness, will not easily collapse imbalance. Organization of ecological chain and biological and ecological chain have different approaches but equally satisfactory results, the ecological chain organization from simple to complex development process, to achieve the robustness from weak to strong. System for cleaning, link to collect information on the organization of ecological selection, eventually back to the ecological chain analysis, intelligent feedback, so that the ecological chain can timely adjust and improve the path to ecological chain in the feedback adjustment to network development. In the symbiosis ecological chain, every component of the ecological chain has interdependent relationship, and it can only be win-win if they cooperate with each other in the ecological 
chain, and the stable development of the ecological chain is especially important. Intelligent feedback system provides intuitive and intelligent self-ecological chain optimization process in high intelligence job embeddedness, in every optimization process will assist High intelligence better to promote ecological chain to the direction of network, so that the stability of the ecological chain gradually increased.

\subsection{The Role of Multiple Variables to Promote High Intelligence Work Embedded Game Equilibrium Dynamic}

Role diversification is the demand of modern development, and the high complex ability of high intelligence in the process of job embeddedness makes it possible to diversify the role of high intelligence in the ecological chain. In the game equilibrium ecological chain, the introduction of high intelligence will lead to the competitive advantage of the game side to other game players. However, under the premise of multiple roles, the high intelligence can be added to many games, which results in the fluctuation of the game results in the ecological chain. The ecological chain in the equilibrium conditions, all participants in the game are satisfied with the results, will let the power lost in the ecological chain of the game, the game equilibrium state for a long time will make the ecological chain lost vitality, but the game party occupy absolute advantage will lead to imbalance of ecological chain collapse. High intellectual role diversification, job embeddedness can make the ecological chain in the game equilibrium, dynamic state.

\subsection{Information Cluster Construction, High Intelligence Work, Embedded Information, Aggregation, Matrix}

According to certain similarity, information clustering refers to the classification and collection of information. In the big data age of massive growth of data, the information cluster can preliminarily process the data. The biggest problem encountered in the eco chain of high intelligence work, embedded in the information aggregation drive, is how to handle data efficiently and accurately. The information cluster makes the information no longer simple linear arrangement, but presents the crisscross arrangement way. This arrangement is flexible and flexible, and can be clustered with different information search requirements. The requirement of information is clear and clear, and the difficulty of processing data with high intelligence is reduced.

\subsection{Multiple Drivers to Create High Intelligence Work, Embedded Intelligent Environment, Ecological}

Multiple drivers are more than one driver core. Multiple driving situation: the development of the ecological chain drive more security, development of the ecological chain does not rely solely on a driving force, high intelligence work in multiple embedded ecological chain drive element complement each other, to avoid the phenomenon; three-dimensional driven, development level is more abundant, the formation and development direction of diversification, can to achieve consumption and output coordination in the ecological chain, to achieve organizational ecology. The multiple driving elements complement each other, and the intelligent alternating cycle promotes the ecological development.

\section{Conclusion}

This paper analyzes the essence and represent of the high intelligence work. We discuss its operation mode embedded the ecosystem in big data era through studying coopetition, value hyperplasia, mutualism, Game equilibrium, information aggregation, environment optimization.

\section{Acknowledgement}

This work is supported by National Social Science Foundation of China" Research on high intelligence value identification and job embeddedness feedback tracking model in big data Era" (No. 15BGL101), Six talent peaks project in Jiangsu Province"Research on high intelligence value identification and job embeddedness feedback tracking model in Internet + Era"(No. JY-030), and Young Academic Leaders Funded of Qing Lan Project of Jiangsu Province.

\section{References}

Charlier, Steven D., Guay, Russell P., \& Zimmerman, Ryan D. (2016). Plugged In or Disconnected? A Model of the Effects of Technological Factors on Employee Job Embeddedness. Human Resource Management, 55(1), 109-126. https://doi.org/10.1002/hrm.21716

Chen Yongqiang, Song Ge \& Zhang Wenjing. (2014). Study on the relationship between job embeddedness and turnover intention of expatriate managers in China. Journal of engineering management, 12, 139-144. 
Collins, Brian J., \& Mossholder, Kevin W. (2017). Fairness Means More to Some than Others: Interactional Fairness, Job Embeddedness, and Discretionary Work Behaviors. Journal of Management, 43(2), 293-318. https://doi.org/10.1002/hrm.21716

Cui Xiao Wei \& Wu Xinnian. (2016). Big data applications for large data industry landing. Science and technology management research, 2, 203-207.

Ferreira, Aristides I., Martinez, Luis F., Lamelas, José Pereira, Rodrigues, \& Rosa I. (2015). Mediation of job embeddedness and satisfaction in the relationship between task characteristics and turnover. Vocational Rehabilitation Services, 118-126.

Li Xin \& Li Na. (2015). Research on the development and innovation ability of high-end talents in China. Science and technology management research, 6, 81-84.

Lou CE Qun, Yu Jie \& Nie Ying. (2015). Structural optimization strategy of network information ecological chain. Library and Information Service, 6-11.

Shen Mengling. (2015). The influence mechanism of job embeddedness analysis. China Management Informationization, 18, 94-94.

Wang Quangang \& Zhao Yongle. (2017). Research on factors affecting global high-end talent flow and agglomeration. Science and technology management research, 91-94.

Xu Xiaojun, Wang Yuting \& Zhang Haitao. (2015). Study on the formation mechanism of business information network ecological chain. Journal of Intelligence, 12, 159-164.

Yang Chunjiang \& Li Ran. (2014). Research on the relationship between organizational ethical climate and employee turnover behavior from the perspective of job embeddedness. Chinese Journal of Management, 2014(3), 351-359.

Yang Tingfang. (2015). Organizational embeddedness, community embeddedness, organizational support, perception and turnover intention. Contemporary Finance \& Economics, 2015(6), 351-360.

Yin Fengchun. (2015). Study on embedded value identification of high-end young talents. Science \& Technology Progress and Policy, 24, 138-142.

Yin Fengchun. (2017). Research on introduction, training mechanism and management innovation of top talents. People's Publishing House.

Zhang Haitao, Sun Hongfei, Sun Siyang \& Song Tuo. (2016). Study on optimization model of business network information ecology. Library and Information Service, 3, 110-117.

Zheng Weibo. (2016). Talent turnover and retention from the perspective of matching: a review of research. Human Resources Development of China, 48-55. 